\title{
EQUIVALENCE AND STABILITY OF RANDOM FIXED POINT ITERATIVE PROCEDURES
}

\author{
ISMAT BEG AND MUJAHID ABBAS
}

Received 21 October 2004; Revised 18 February 2005; Accepted 2 March 2005

We generate a sequence of measurable mappings iteratively and study necessary conditions for its strong convergence to a random fixed point of strongly pseudocontractive random operator. We establish the weak convergence of an implicit random iterative procedure to common random fixed point of a finite family of nonexpansive random operators in Hilbert spaces. We prove the equivalence between the convergence of random Ishikawa and random Mann iterative schemes for contraction random operator and strongly pseudocontractive random operator. We also examine the stability of random fixed point iterative procedures for the random operators satisfying certain contractive conditions in the context of metric spaces.

Copyright (c) 2006 I. Beg and M. Abbas. This is an open access article distributed under the Creative Commons Attribution License, which permits unrestricted use, distribution, and reproduction in any medium, provided the original work is properly cited.

\section{Introduction}

Random nonlinear analysis is an important mathematical discipline which is mainly concerned with the study of random nonlinear operators and its development is required for the study of various classes of random operator equations. The systematic study of random operator equations employing the methods of functional analysis was first initiated by the Prague school of probabilists in the 1950s. The study of random fixed points is the core around which the theory of random operators has developed. Random fixed point theorems for random contraction mappings on separable complete metric spaces were first proved by Špaček [24] and Hanš (see, [12, 13]). The survey article by BharuchaReid [6] in 1976 attracted the attention of several mathematicians and gave wings to this theory. Itoh [14] extended Špaček's and Hanšs theorems to multivalued contraction mappings. Now this theory has become the full-fledged research area and various ideas associated with random fixed point theory are applied to obtain the solutions to a class of stochastic integral equations (see [19]). Recently, Beg [2, 3], Beg and Shahzad [4, 5], Lin [16], Papageorgiou [20], Tan and Yuan [26], Xu [28], Xu and Beg [29], and many other authors have studied the stochastic solvability of the random operator equation 
and its various applications in diverse areas from pure mathematics to applied sciences. The aim of this paper is to study the behaviour of the sequence of measurable mappings constructed through random Ishikawa and random Mann iterative procedures involving strongly pseudocontractive random operators in Banach spaces. We construct an implicit random iterative scheme and study its weak convergence to common random fixed point of finite family of nonexpansive random operators in the context of Hilbert spaces. We prove that the convergences of random Ishikawa and random Mann iterative procedures for contraction random operators and strongly pseudocontractive random operators are equivalent. We also examine the stability of random fixed point iterative procedures for the random operators satisfying certain contractive conditions in the context of metric spaces.

\section{Preliminaries}

Let $(\Omega, \Sigma)$ be a measurable space ( $\Sigma$-sigma algebra) and $F$ a nonempty subset of a Banach space $X$. A mapping $\xi: \Omega \rightarrow X$ is measurable if $\xi^{-1}(U) \in \Sigma$, for each open subset $U$ of $X$. The mapping $T: \Omega \times F \rightarrow F$ is a random map if and only if for each fixed $x \in F$, the mapping $T(\cdot, x): \Omega \rightarrow F$ is measurable and it is continuous if for each $\omega \in \Omega$, the mapping $T(\omega, \cdot): F \rightarrow X$ is continuous. A measurable mapping $\xi: \Omega \rightarrow X$ is the random fixed point of the random map $T: \Omega \times F \rightarrow X$ if and only if it is the stochastic solution of random operator equation $I-T(\omega, x)=x$, for each $\omega \in \Omega$ and $x \in F$; the letter $I$ denotes the random mapping $I: \Omega \times F \rightarrow F$ defined by $I(\omega, x)=x$. We denote the set of random fixed points of a random map $T$ by $\mathrm{RF}(T)$.

Let $B\left(x_{0}, r\right)$ denote the spherical ball centred at $x_{0}$ with radius $r$, defined as the set $\left\{x \in X:\left\|x-x_{0}\right\| \leq r\right\}$.

We denote the $n$th iterate $T(\omega, T(\omega, T(\omega, \ldots, T(\omega, x), \ldots)))$ of $T$ by $T^{n}(\omega, x)$ and $T^{0}=I$.

Definition 2.1. Let $F$ be a nonempty subset of a separable Banach space $X$ and let $T$ : $\Omega \times F \rightarrow F$ be a random map.

(a) The map $T$ is said to be an asymptotically nonexpansive random operator if there exists a sequence of measurable mappings $k_{n}: \Omega \rightarrow[1, \infty)$ with $\lim _{n \rightarrow \infty} k_{n}(\omega)=1$, for each $\omega \in \Omega$, such that for arbitrary $x, y \in F$,

$$
\left\|T^{n}(\omega, x)-T^{n}(\omega, y)\right\| \leq k_{n}(\omega)\|x-y\|, \quad \text { for each } \omega \in \Omega .
$$

Taking $k_{n}(\omega)=1$, for every $\omega \in \Omega$ and for every $n \in \mathbb{N}$, replacing $n$th iterate of $T$ by $T$, there exists a nonexpansive random operator. It is known that nonexpansive random operator from $\Omega \times F \rightarrow F$ has a random fixed point when $F$ is closed convex and bounded subset of a separable Banach space $X$ satisfying Opial's condition.

(b) The map $T$ is said to be a uniformly L-Lipschitzian random operator if for arbitrary $x, y \in F$,

$$
\left\|T^{n}(\omega, x)-T^{n}(\omega, y)\right\| \leq L\|x-y\|, \quad \text { for each } \omega \in \Omega,
$$

where $n=1,2, \ldots$, and $L$ is a positive constant. Replacing $n$th iterate of $T$ by the random operator $T$, the definition of $L$-Lipschitzian random operator is obtained. An asymptotically nonexpansive random operator is uniformly $L$-Lipschitzian random operator for some $L \geq 1$. 
(c) The map $T$ is said to be a pseudocontractive random operator if for any measurable mapping $r: \Omega \rightarrow(0, \infty)$ and for arbitrary $x, y \in F$,

$$
\|x-y\| \leq\|(1+r(\omega))(x-y)-r(\omega)(T(\omega, x)-T(\omega, y))\|,
$$

for each $\omega \in \Omega$. This class of random operators is more general than the class of nonexpansive random operators. The importance of this class stems from the connection which exists between pseudocontractive random operators and assertive random operators; namely, $T(\omega, \cdot)$ is pseudocontractive random operator if and only if $I-T(\omega, \cdot)$ is assertive random operator, for each $\omega \in \Omega$.

(d) The map $T$ is said to be a $k(\omega)$-strongly pseudocontractive random operator for some measurable mapping $k: \Omega \rightarrow(0,1)$ if for any measurable mapping $r: \Omega \rightarrow(0, \infty)$ and for each $x \in F$,

$$
\|x-y\| \leq k(\omega)\|1+r(\omega)(x-y)-r(\omega)(T(\omega, x)-T(\omega, y))\|, \quad y \in F,
$$

for each $\omega \in \Omega$. $F$,

(e) The map $T$ is said to be a weakly contractive random operator if for arbitrary $x, y \in$

$$
\|T(\omega, x)-T(\omega, y)\| \leq\|x-y\|-\Psi(\|x-y\|), \quad \text { for each } \omega \in \Omega,
$$

where $\Psi:[0, \infty) \rightarrow[0, \infty)$ is a continuous and nondecreasing map such that $\Psi(0)=0$, and $\lim _{t \rightarrow \infty} \Psi(t)=\infty$.

If $\Psi(t)=(1-k(\omega)) t$, where $k: \Omega \rightarrow(0,1)$ is any fixed measurable mapping, then the weakly contractive random operator becomes a $k(\omega)$-contraction random operator and it has a unique random fixed point, according to Bharucha-Reid [6].

Definition 2.2 (random Mann iterative process). Let $T: \Omega \times F \rightarrow F$ be a random operator, where $F$ is a nonempty closed and convex subset of a separable Banach space $X$. Then random Mann iterative process is the sequence of functions $\left\{\xi_{n}\right\}$ defined by

$$
\xi_{n+1}(\omega)=\left(1-\alpha_{n}(\omega)\right) \xi_{n}(\omega)+\alpha_{n}(\omega) T\left(\omega, \xi_{n}(\omega)\right), \quad \text { for each } \omega \in \Omega
$$

$n=0,1,2, \ldots$, where $\alpha_{n}: \Omega \rightarrow[0,1]$ is measurable mappings for each $n \in \mathbb{N}$ and $\xi_{0}: \Omega \rightarrow F$ is an arbitrary fixed measurable mapping. Obviously $\left\{\xi_{n}\right\}$ is a sequence of functions from $\Omega$ to $F$.

Remark 2.3. Let $F$ be a closed and convex subset of a separable Banach space $X$ and let the sequence of functions $\left\{\xi_{n}\right\}$ defined as in Definition 2.2 be pointwise convergent, that is, $\xi_{n}(\omega) \rightarrow q:=\xi(\omega)$, for each $\omega \in \Omega$. Then closedness of $F$ implies that $\xi$ is a mapping from $\Omega$ to $F$. Since $F$ is a subset of a separable Banach space $X$, so, if $T$ is a continuous random operator, then by $[1$, Lemma 8.2.3] the map $\omega \rightarrow T(\omega, f(\omega))$ is a measurable function for any measurable function $f$ from $\Omega$ to $F$. Thus $\left\{\xi_{n}\right\}$ is a sequence of measurable functions. Hence $\xi: \Omega \rightarrow F$, being the limit of the sequence of measurable functions, is also measurable. 
4 Equivalence of random iterative procedure

Definition 2.4 (random Ishikawa iterative process). Let $T: \Omega \times F \rightarrow F$ be a random operator, where $F$ is a nonempty closed and convex subset of a separable Banach space $X$. Then random Ishikawa iterative process is the sequences of functions $\left\{\xi_{n}\right\}$ and $\left\{\eta_{n}\right\}$ defined by

$$
\begin{gathered}
\xi_{n+1}(\omega)=\left(1-\alpha_{n}(\omega)\right) \xi_{n}(\omega)+\alpha_{n}(\omega) T\left(\omega, \eta_{n}(\omega)\right), \\
\eta_{n}(\omega)=\left(1-\beta_{n}(\omega)\right) \xi_{n}(\omega)+\beta_{n}(\omega) T\left(\omega, \xi_{n}(\omega)\right), \quad \text { for each } \omega \in \Omega,
\end{gathered}
$$

$n=0,1,2, \ldots$, where $\alpha_{n}$ and $\beta_{n}: \Omega \rightarrow[0,1]$ are measurable mappings for each $n \in \mathbb{N}$ and $\xi_{0}: \Omega \rightarrow F$ is an arbitrary measurable mapping. Obviously $\left\{\xi_{n}\right\}$ and $\left\{\eta_{n}\right\}$ are sequences of measurable functions from $\Omega$ to $F$. Taking $\beta_{n}(\omega)=0$, for each $\omega \in \Omega$, the random Mann iterative process is obtained.

Definition 2.5 (random Kirk iteration scheme). Let $T: \Omega \times F \rightarrow F$ be a random operator, where $F$ is a nonempty closed and convex subset of a separable Banach space $X$. Then, for a fixed positive integer $k$, define the map $S: \Omega \times F \rightarrow F$ as

$$
S(\omega, x)=\sum_{i=0}^{k} \alpha_{i}(\omega) T^{i}(\omega, x), \quad \text { for each } \omega \in \Omega,
$$

where $\alpha_{i}(\omega) \geq 0, \sum_{i=0}^{k} \alpha_{i}(\omega)=1$, and $\alpha_{1}(\omega) \neq 0$, for each $\omega \in \Omega$. Now the sequence of functions $\left\{\xi_{n}\right\}$ given by the following equation defines random Kirk iterative process:

$$
\xi_{n+1}(\omega)=S\left(\omega, \xi_{n}(\omega)\right), \quad \text { for each } \omega \in \Omega, n=0,1,2, \ldots,
$$

where $\xi_{0}: \Omega \rightarrow F$ is an arbitrary fixed measurable mapping. Obviously $\left\{\xi_{n}\right\}$ is a sequence of functions from $\Omega$ to $F$.

Definition 2.6. Let $\left\{T_{1}, T_{2}, T_{3}, \ldots, T_{\mathbb{N}}\right\}$ be a family of random operators from $\Omega \times F$ to $F$, where $F$ is a closed, bounded, and convex subset of a separable Banach space $X$. Let $D=\bigcap_{i=1}^{\mathbb{N}} \operatorname{RF}\left(T_{i}\right) \neq \phi$, where $\operatorname{RF}\left(T_{i}\right)$ is the set of all random fixed points of a random operator $T_{i}$, for each $i \in\{1,2,3, \ldots, \mathbb{N}\}=J$. Let $\xi_{0}: \Omega \rightarrow F$ be any fixed measurable map. Let $\alpha_{n}: \Omega \rightarrow(0,1)$ be measurable mappings for each $n \in \mathbb{N}$ and $\lim _{n \rightarrow \infty} \alpha_{n}(\omega)=0$ for each $\omega \in \Omega$. The sequence of functions $\left\{\xi_{n}\right\}$ is defined as follows:

$$
\begin{gathered}
\xi_{1}(\omega)=\alpha_{1}(\omega) \xi_{0}(\omega)+\left(1-\alpha_{1}(\omega)\right) T_{1}\left(\omega, \xi_{1}(\omega)\right), \\
\xi_{2}(\omega)=\alpha_{2}(\omega) \xi_{1}(\omega)+\left(1-\alpha_{2}(\omega)\right) T_{2}\left(\omega, \xi_{2}(\omega)\right), \\
\vdots \\
\xi_{\mathbb{N}}(\omega)=\alpha_{\mathbb{N}}(\omega) \xi_{\mathbb{N}-1}(\omega)+\left(1-\alpha_{\mathbb{N}}(\omega)\right) T_{\mathbb{N}}\left(\omega, \xi_{\mathbb{N}}(\omega)\right), \\
\xi_{\mathbb{N}+1}(\omega)=\alpha_{\mathbb{N}+1}(\omega) \xi_{\mathbb{N}}(\omega)+\left(1-\alpha_{\mathbb{N}+1}(\omega)\right) T_{1}\left(\omega, \xi_{\mathbb{N}+1}(\omega)\right), \\
\vdots \\
\xi_{2 \mathbb{N}}(\omega)=\alpha_{2 \mathbb{N}}(\omega) \xi_{2 \mathbb{N}-1}(\omega)+\left(1-\alpha_{2 \mathbb{N}}(\omega)\right) T_{\mathbb{N}}\left(\omega, \xi_{2 \mathbb{N}}(\omega)\right), \\
\xi_{2 \mathbb{N}+1}(\omega)=\alpha_{2 \mathbb{N}+1}(\omega) \xi_{2 \mathbb{N}}(\omega)+\left(1-\alpha_{2 \mathbb{N}+1}(\omega)\right) T_{1}\left(\omega, \xi_{2 \mathbb{N}+1}(\omega)\right),
\end{gathered}
$$


In compact form, we have

$$
\xi_{n}(\omega)=\alpha_{n}(\omega) \xi_{n-1}(\omega)+\left(1-\alpha_{n}(\omega)\right) T_{n}\left(\omega, \xi_{n}(\omega)\right), \quad n \geq 1,
$$

where $T_{n}=T_{n \bmod \mathbb{N}}$.

Definition 2.7. Let $T: \Omega \times F \rightarrow F$ be a random operator, where $F$ is a nonempty closed and convex subset of a separable Banach space $X$. Let $\xi_{0}: \Omega \rightarrow F$ be any fixed measurable mapping. The sequence $\left\{\xi_{n+1}(\omega)\right\}$ of measurable mappings from $\Omega$ to $F$, for $n=0,1,2, \ldots$, generated by the certain iterative procedure involving a random operator $T$ is denoted by $\left\{T, \xi_{n}(\omega)\right\}$, for each $\omega \in \Omega$. Suppose $\xi_{n+1}(\omega) \rightarrow \xi^{*}(\omega)$ as $n \rightarrow \infty$, for each $\omega \in \Omega$, where $\xi^{*} \in \operatorname{RF}(T)$. Let $\left\{\eta_{n}\right\}$ be any sequence of measurable mappings from $\Omega$ to $F$. Define the sequence of measurable mappings $k_{n}: \Omega \rightarrow R$ by $k_{n}(\omega)=d\left(\eta_{n}(\omega),\left\{T, \eta_{n}(\omega)\right\}\right)$ (measurability of $k_{n}$ follows from [1, Corollary 8.2.13]). If for each $\omega \in \Omega, k_{n}(\omega) \rightarrow 0$, as $n \rightarrow \infty$ implies $\eta_{n}(\omega) \rightarrow \xi^{*}(\omega)$ as $n \rightarrow \infty$, for each $\omega \in \Omega$, then a random iterative procedure is said to be stable with respect to random operator $T$.

Definition 2.8. Let $F$ be a nonempty closed and convex subset of a Banach space $X$. A mapping $T: F \rightarrow X$ is called demiclosed with respect to $y \in X$ for each sequence $\left\{x_{n}\right\}$ in $F$ such that $\left\{x_{n}\right\}$ converges weakly to $x \in X$ and $\left\{T x_{n}\right\}$ converges strongly to $y$ implying that $x \in F$ and $T x=y$.

Let $F$ be a closed and convex subset of a Hilbert space $H$ and let $T: F \rightarrow H$ be a nonexpansive mapping then $I-T$ is demiclosed on $F$. A Banach space $X$ has Opial property if $x_{n} \rightarrow x$ weakly implies that $\limsup _{n}\left\|x_{n}-x\right\|<\limsup _{n}\left\|x_{n}-x\right\|$ for any $z \in X, z \neq x$. Banach spaces satisfying Opial property include Hilbert spaces and $l^{p}(1<p<\infty)$ spaces while $L_{p}$ spaces $(p \neq 2)$ are not Opial spaces. For further details and other related results we refer to [18].

A random operator $T: \Omega \times F \rightarrow F$ is called demiclosed if $T(\omega, \cdot)$ is demiclosed for each $\omega \in \Omega$.

Definition 2.9. Let $X$ and $Y$ be two separable Banach spaces. Let $F$ be a nonempty weakly compact subset of $X$. A continuous random operator $T: \Omega \times F \rightarrow Y$ is said to satisfy the Leray-Schauder boundary condition if for each $\omega \in \Omega$, there exists $z \in \operatorname{int}(F)$ (depending on $\omega)$ such that

$$
T(\omega, y)-z \neq \alpha(y-z) \quad \forall y \in \partial(F), \alpha>1,
$$

where $\partial(F)$ stands for the boundary of $F$.

We will also need the following lemma from [27].

LEMMA 2.10. Let the nonnegative number sequence $\left\{\alpha_{n}\right\}$ satisfy that

$$
\alpha_{n+1} \leq\left(1-\delta_{n}\right) \alpha_{n}+\sigma_{n}, \quad \text { for each } n=1,2,3, \ldots ; \delta_{n} \in[0,1], \sum_{i=1}^{\infty} \delta_{i}=\infty
$$

and $\sigma_{n}=o\left(\delta_{n}\right)$. Then, $\lim _{n \rightarrow \infty} \alpha_{n}=0$. 


\section{Convergence and equivalence of random Mann and random Ishikawa iterative schemes for strongly pseudocontractive random operator}

The Ishikawa and Mann iterative schemes have been successfully applied for obtaining the solutions of deterministic operator equations. Recently, Chidume [7, 8], Górnicki [11], Park [21], Singh and Watson [23], and Tan and Xu [25] have used different iteration procedures to obtain fixed points in deterministic operator theory while Kirk [15], Gatica and Kirk [9], and Goebel and Kirk [10] have studied the existence of fixed points of asymptotically nonexpansive and pseudocontractive mappings, the class of mappings more general than nonexpansive mappings. In this section, assuring the existence of random fixed point of strongly pseudocontractive random operator, the convergence of random Mann and random Ishikawa schemes to the random fixed point of strongly pseudocontractive random operator is proved and equivalence of these two random iterative procedures is also established. First, we present the following convergence theorem.

Theorem 3.1. Let $F$ be a nonempty closed bounded and convex subset of a separable reflexive Banach space $X$ with $0 \in \operatorname{int}(F)$. Let $T: \Omega \times F \rightarrow F$ be an L-Lipschitz and strongly pseudocontractive random operator satisfying the Leray-Schauder boundary condition for $z=0$. Let $\xi_{0}$ be a fixed measurable mapping from $\Omega$ to $F$. Define the sequences of functions $\left\{\eta_{n}\right\}$ and $\left\{\xi_{n}\right\}$ as given below:

$$
\begin{gathered}
\eta_{n}(\omega)=\beta_{n}(\omega) T\left(\omega, \xi_{n}(\omega)\right)+\left(1-\beta_{n}(\omega)\right) \xi_{n}(\omega), \\
\xi_{n+1}(\omega)=\alpha_{n}(\omega) T\left(\omega, \eta_{n}(\omega)\right)+\left(1-\alpha_{n}(\omega)\right) \xi_{n}(\omega), \quad \text { for each } \omega \in \Omega,
\end{gathered}
$$

$n=0,1,2, \ldots$, where $\alpha_{n}$ and $\beta_{n}: \Omega \rightarrow[0,1]$ are measurable mappings for each $n \in \mathbb{N}$, $\sum_{n \geq 0} \beta_{n}(\omega)$ and $\sum_{n \geq 0} \alpha_{n}(\omega)<\infty$, for each $\omega \in \Omega$. Then the sequence of measurable mappings $\left\{\xi_{n}\right\}$ generated iteratively converges strongly to the random fixed point of $T$.

Proof. For every $\omega \in \Omega$, we have

$$
\begin{aligned}
\xi_{n}(\omega)= & \left(1+\alpha_{n}(\omega)\right) \xi_{n+1}(\omega)+\alpha_{n}(\omega)\left(\xi_{n}(\omega)-\xi_{n+1}(\omega)\right)-\alpha_{n}(\omega) T\left(\omega, \eta_{n}(\omega)\right), \\
\xi_{n}(\omega)= & \left(1+\alpha_{n}(\omega)\right) \xi_{n+1}(\omega)+\alpha_{n}(\omega)\left(\alpha_{n}(\omega) \xi_{n}(\omega)-\alpha_{n}(\omega) T\left(\omega, \eta_{n}(\omega)\right)\right) \\
& -\alpha_{n}(\omega)\left(T\left(\omega, \eta_{n}(\omega)\right)-T\left(\omega, \xi_{n+1}(\omega)\right)\right)-\alpha_{n}(\omega) T\left(\omega, \xi_{n+1}(\omega)\right) .
\end{aligned}
$$

Let $\xi^{*}: \Omega \rightarrow F$ be the random fixed point of $T$, the existence of random fixed point of $T$ follows from [22, Theorem 3.10]. Therefore,

$$
\xi^{*}(\omega)=\left(1+\alpha_{n}(\omega)\right) \xi^{*}(\omega)-\alpha_{n}(\omega) T\left(\omega, \xi^{*}(\omega)\right),
$$

for every $\omega \in \Omega$. Now consider for $\omega \in \Omega$,

$$
\begin{aligned}
\xi_{n}(\omega)-\xi^{*}(\omega)= & 1+\alpha_{n}(\omega)\left(\xi_{n+1}(\omega)-\xi^{*}(\omega)\right) \\
& +\alpha_{n}(\omega)\left(T\left(\omega, \xi^{*}(\omega)\right)-T\left(\omega, \xi_{n+1}(\omega)\right)\right) \\
& -\alpha_{n}(\omega)\left(T\left(\omega, \eta_{n}(\omega)\right)-T\left(\omega, \xi_{n+1}(\omega)\right)\right) \\
& -\alpha_{n}(\omega)\left(\alpha_{n}(\omega) T\left(\omega, \eta_{n}(\omega)\right)-\alpha_{n}(\omega) \xi_{n}(\omega)\right) .
\end{aligned}
$$


Since $T$ is strongly pseudocontractive random operator, so taking the norm of the sum of the first two terms on the right-hand side of (3.4), we obtain

$$
\begin{gathered}
\left\|\left(1+\alpha_{n}(\omega)\right)\left(\xi_{n+1}(\omega)-\xi^{*}(\omega)\right)+\alpha_{n}(\omega)\left(T\left(\omega, \xi^{*}(\omega)\right)-T\left(\omega, \xi_{n+1}(\omega)\right)\right)\right\| \\
\geq\left\|\xi_{n+1}(\omega)-\xi^{*}(\omega)\right\|, \quad \text { for every } \omega \in \Omega .
\end{gathered}
$$

Thus,

$$
\begin{aligned}
\left\|\xi_{n}(\omega)-\xi^{*}(\omega)\right\| \geq & \left\|\xi_{n+1}(\omega)-\xi^{*}(\omega)\right\|-\alpha_{n}(\omega)\left\|T\left(\omega, \eta_{n}(\omega)\right)-T\left(\omega, \xi_{n+1}(\omega)\right)\right\| \\
& -\alpha_{n}(\omega)\left\|\alpha_{n}(\omega) \xi_{n}(\omega)-\alpha_{n}(\omega) T\left(\omega, \eta_{n}(\omega)\right)\right\|, \quad \text { for every } \omega \in \Omega
\end{aligned}
$$

which further implies

$$
\begin{aligned}
\left\|\xi_{n+1}(\omega)-\xi^{*}(\omega)\right\| \leq & \left\|\xi_{n}(\omega)-\xi^{*}(\omega)\right\|+\alpha_{n}(\omega)\left\|T\left(\omega, \eta_{n}(\omega)\right)-T\left(\omega, \xi_{n+1}(\omega)\right)\right\| \\
& +\alpha_{n}(\omega)\left\|\alpha_{n}(\omega) \xi_{n}(\omega)-\alpha_{n}(\omega) T\left(\omega, \eta_{n}(\omega)\right)\right\|, \quad \text { for every } \omega \in \Omega .
\end{aligned}
$$

Now,

$$
\begin{aligned}
\left\|\xi_{n}(\omega)-T\left(\omega, \xi_{n}(\omega)\right)\right\| & \leq\left\|\xi_{n}(\omega)-\xi^{*}(\omega)\right\|+\left\|T\left(\omega, \xi^{*}(\omega)\right)-T\left(\omega, \xi_{n}(\omega)\right)\right\| \\
& \leq\left\|\xi_{n}(\omega)-\xi^{*}(\omega)\right\|+L\left\|\xi_{n}(\omega)-\xi^{*}(\omega)\right\| \\
& \leq(1+L)\left\|\xi_{n}(\omega)-\xi^{*}(\omega)\right\|, \quad \text { for every } \omega \in \Omega .
\end{aligned}
$$

Also,

$$
\begin{aligned}
\| T(\omega, & \left.\xi_{n}(\omega)\right)-T\left(\omega, \eta_{n}(\omega)\right) \| \\
& \leq L\left\|\xi_{n}(\omega)-\eta_{n}(\omega)\right\| \\
& \leq L\left\|\xi_{n}(\omega)-\left(1-\beta_{n}(\omega)\right) \xi_{n}(\omega)-\beta_{n}(\omega) T\left(\omega, \xi_{n}(\omega)\right)\right\| \\
& \leq L \beta_{n}(\omega)\left\|\xi_{n}(\omega)-T\left(\omega, \xi_{n}(\omega)\right)\right\| \\
& \leq L \beta_{n}(\omega)(1+L)\left\|\xi_{n}(\omega)-\xi^{*}(\omega)\right\|, \quad \text { for every } \omega \in \Omega, \\
\left\|\eta_{n}(\omega)-\xi^{*}(\omega)\right\| & \left\|\left(1-\beta_{n}(\omega)\right) \xi_{n}(\omega)+\beta_{n}(\omega) T\left(\omega, \xi_{n}(\omega)\right)-\xi^{*}(\omega)\right\| \\
& =\left\|1-\beta_{n}(\omega)\left(\xi_{n}(\omega)-\xi^{*}(\omega)\right)+\beta_{n}(\omega)\left(T\left(\omega, \xi_{n}(\omega)\right)-\xi^{*}(\omega)\right)\right\| \\
& \leq 1-\beta_{n}(\omega)\left\|\xi_{n}(\omega)-\xi^{*}(\omega)\right\|+\beta_{n}(\omega)\left\|T\left(\omega, \xi_{n}(\omega)\right)-\xi^{*}(\omega)\right\| \\
& \leq 1-\beta_{n}(\omega)\left\|\xi_{n}(\omega)-\xi^{*}(\omega)\right\|+\beta_{n}(\omega) L\left\|\xi_{n}(\omega)-\xi^{*}(\omega)\right\| \\
& \leq 1-\beta_{n}(\omega)+\beta_{n}(\omega) L\left\|\xi_{n}(\omega)-\xi^{*}(\omega)\right\|, \quad \text { for every } \omega \in \Omega .
\end{aligned}
$$


8 Equivalence of random iterative procedure

Now,

$$
\begin{aligned}
\left\|\xi_{n}(\omega)-T\left(\omega, \eta_{n}(\omega)\right)\right\| & \leq\left\|\xi_{n}(\omega)-\xi^{*}(\omega)\right\|+\left\|\xi^{*}(\omega)-T\left(\omega, \eta_{n}(\omega)\right)\right\| \\
& \leq\left\|\xi_{n}(\omega)-\xi^{*}(\omega)\right\|+L\left\|\xi^{*}(\omega)-\eta_{n}(\omega)\right\| \\
& \leq\left\|\xi_{n}(\omega)-\xi^{*}(\omega)\right\|+L\left(1-\beta_{n}(\omega)+\beta_{n}(\omega) L\right)\left\|\xi_{n}(\omega)-\xi^{*}(\omega)\right\| \\
& \leq 1+L\left(1-\beta_{n}(\omega)+\beta_{n}(\omega) L\right)\left\|\xi_{n}(\omega)-\xi^{*}(\omega)\right\| \\
& =1+L+\beta_{n}(\omega) L(L-1)\left\|\xi_{n}(\omega)-\xi^{*}(\omega)\right\|,
\end{aligned}
$$

for each $\omega \in \Omega$. Finally, we have

$$
\begin{aligned}
& \left\|T\left(\omega, \eta_{n}(\omega)\right)-T\left(\omega, \xi_{n+1}(\omega)\right)\right\| \\
& \quad \leq L\left\|\eta_{n}(\omega)-\xi_{n+1}(\omega)\right\| \\
& \quad=L\left\|\left(1-\beta_{n}(\omega)\right) \xi_{n}(\omega)+\beta_{n}(\omega) T\left(\omega, \xi_{n}(\omega)\right)-\left(1-\alpha_{n}(\omega)\right) \xi_{n}(\omega)-\alpha_{n}(\omega) T\left(\omega, \eta_{n}(\omega)\right)\right\| \\
& \quad=L\left\|\beta_{n}(\omega)\left(T\left(\omega, \xi_{n}(\omega)\right)-\xi_{n}(\omega)\right)+\alpha_{n}(\omega)\left(\xi_{n}(\omega)-T\left(\omega, \eta_{n}(\omega)\right)\right)\right\| \\
& \quad \leq L\left(\beta_{n}(\omega)\left\|T\left(\omega, \xi_{n}(\omega)\right)-\xi_{n}(\omega)\right\|+\alpha_{n}(\omega)\left\|\xi_{n}(\omega)-T\left(\omega, \eta_{n}(\omega)\right)\right\|\right) \\
& \quad \leq L\left(\beta_{n}(\omega)(1+L)\left\|\xi_{n}(\omega)-\xi^{*}(\omega)\right\|+\alpha_{n}(\omega)\left(1+L+\beta_{n}(\omega) L(L-1)\right)\left\|\xi_{n}(\omega)-\xi^{*}(\omega)\right\|\right) \\
& \quad \leq L\left(\beta_{n}(\omega)(1+L)+\alpha_{n}(\omega)\left(1+L+\beta_{n}(\omega) L(L-1)\right)\right)\left\|\xi_{n}(\omega)-\xi^{*}(\omega)\right\|
\end{aligned}
$$

for every $\omega \in \Omega$. Now inequality (3.7) becomes

$$
\begin{aligned}
\left\|\xi_{n+1}(\omega)-\xi^{*}(\omega)\right\| \leq & 1+\alpha_{n}(\omega)\left(1+L+\beta_{n}(\omega) L(L-1)\right) \\
& +L\left(\beta_{n}(\omega)(1+L)+\alpha_{n}(\omega)\left(1+L+\beta_{n}(\omega) L(L-1)\right)\right)\left\|\xi_{n}(\omega)-\xi^{*}(\omega)\right\|,
\end{aligned}
$$

for every $\omega \in \Omega$. Thus,

$$
\left\|\xi_{n+1}(\omega)-\xi^{*}(\omega)\right\| \leq 1+\delta_{n}(\omega)\left\|\xi_{n}(\omega)-\xi^{*}(\omega)\right\|, \quad \text { for every } \omega \in \Omega
$$

where $\delta_{n}(\omega)=\alpha_{n}(\omega)\left(1+L+\beta_{n}(\omega) L(L-1)\right)+L\left(\beta_{n}(\omega)(1+L)+\alpha_{n}(\omega)\left(1+L+\beta_{n}(\omega) L(L-\right.\right.$ $1)))$. Applying [17, Lemma 2$]$, we obtain $\lim _{n \rightarrow \infty}\left\|\xi_{n}(\omega)-\xi^{*}(\omega)\right\|$, for each $\omega \in \Omega$. Since $T$ is strongly pseudocontractive random operator, therefore $\lim _{n \rightarrow \infty}\left\|\xi_{n}(\omega)-\xi^{*}(\omega)\right\|=0$, for each $\omega \in \Omega$, otherwise it will contradict the choice of the measurable mapping $k: \Omega \rightarrow$ $(0,1)$.

Corollary 3.2. Let $F$ be a nonempty closed bounded and convex subset of a separable reflexive Banach space $X$ with $0 \in \operatorname{int}(F)$. Let $T: \Omega \times F \rightarrow F$ be an L-Lipschitz and strongly pseudocontractive random operator satisfying the Leray-Schauder boundary condition for $z=0$. Let $\xi_{0}$ be a fixed measurable mapping from $\Omega$ to $F$. Define the sequence of functions $\left\{\xi_{n}\right\}$ as given below:

$$
\xi_{n+1}(\omega)=\alpha_{n}(\omega) T\left(\omega, \xi_{n}(\omega)\right)+\left(1-\alpha_{n}(\omega)\right) \xi_{n}(\omega), \quad \text { for each } \omega \in \Omega,
$$


$n=0,1,2, \ldots$, where $\alpha_{n}: \Omega \rightarrow[0,1]$ is measurable mappings for each $n \in \mathbb{N}$, and $\sum_{n \geq 0} \alpha_{n}(\omega)<$ $\infty$, for each $\omega \in \Omega$. Then the sequence of measurable mappings $\left\{\xi_{n}\right\}$ generated iteratively converges strongly to the random fixed point of $T$.

The following theorem establishes a relationship between random Ishikawa iterative and random Mann iterative procedures.

Theorem 3.3. Let $F$ be a nonempty closed bounded and convex subset of a separable reflexive Banach space $X$ with $0 \in \operatorname{int}(F)$. Let $T: \Omega \times F \rightarrow F$ be an L-Lipschitz with $L \geq 1$ and strongly pseudocontractive random operator satisfying the Leray-Schauder boundary condition for $z=0$. Let $\xi_{0}=\zeta_{0}$ be a fixed measurable mapping from $\Omega$ to $F$. Define the sequence of functions $\left\{\eta_{n}\right\}$ and $\left\{\xi_{n}\right\}$ as given below:

$$
\begin{gathered}
\eta_{n}(\omega)=\beta_{n}(\omega) T\left(\omega, \xi_{n}(\omega)\right)+\left(1-\beta_{n}(\omega)\right) \xi_{n}(\omega), \\
\xi_{n+1}(\omega)=\alpha_{n}(\omega) T\left(\omega, \eta_{n}(\omega)\right)+\left(1-\alpha_{n}(\omega)\right) \xi_{n}(\omega), \quad \text { for each } \omega \in \Omega, \\
\zeta_{n+1}(\omega)=\left(1-\alpha_{n}(\omega)\right) \zeta_{n}(\omega)+\alpha_{n}(\omega) T\left(\omega, \zeta_{n}(\omega)\right), \quad \text { for each } \omega \in \Omega,
\end{gathered}
$$

$n=0,1,2, \ldots$, where $\alpha_{n}$ and $\beta_{n}: \Omega \rightarrow[0,1]$ are measurable mappings (same for both random iterative procedures) for each $n \in \mathbb{N}$, and $\lim _{n \rightarrow \infty} \alpha_{n}(\omega)=0=\lim _{n \rightarrow \infty} \beta_{n}(\omega)$ and $\sum_{n \geq 0} \alpha_{n}(\omega)=\infty$, for each $\omega \in \Omega$. Then the sequence of measurable mappings $\left\{\xi_{n}\right\}$ converges strongly to the random fixed point $\xi^{*}$ of $T$ if and only if the sequence of measurable mappings $\left\{\zeta_{n}\right\}$ converges strongly to the random fixed point $\xi^{*}$ of $T$.

Proof. Corollary 3.2 establishes the necessary condition. For the sufficient condition, consider

$$
\begin{aligned}
\xi_{n}(\omega)= & \xi_{n+1}(\omega)+\alpha_{n}(\omega) \xi_{n}(\omega)-\alpha_{n}(\omega) T\left(\omega, \eta_{n}(\omega)\right) \\
= & \left(1+\alpha_{n}(\omega)\right) \xi_{n+1}(\omega)+\alpha_{n}(\omega)\left(\xi_{n+1}(\omega)-T\left(\omega, \xi_{n+1}(\omega)\right)\right) \\
& -2 \alpha_{n}(\omega) \xi_{n+1}(\omega)+\alpha_{n}(\omega) \xi_{n}(\omega)+\alpha_{n}(\omega)\left(T\left(\omega, \xi_{n+1}(\omega)\right)-T\left(\omega, \eta_{n}(\omega)\right)\right) \\
= & \left(1+\alpha_{n}(\omega)\right) \xi_{n+1}(\omega)+\alpha_{n}(\omega)\left(\xi_{n+1}(\omega)-T\left(\omega, \xi_{n+1}(\omega)\right)\right) \\
& -2 \alpha_{n}(\omega)\left(\xi_{n}(\omega)+\alpha_{n}(\omega)\left(T\left(\omega, \eta_{n}(\omega)\right)-\xi_{n}(\omega)\right)\right) \\
& +\alpha_{n}(\omega) \xi_{n}(\omega)+\alpha_{n}(\omega)\left(T\left(\omega, \xi_{n+1}(\omega)\right)-T\left(\omega, \eta_{n}(\omega)\right)\right) \\
= & \left(1+\alpha_{n}(\omega)\right) \xi_{n+1}(\omega)+\alpha_{n}(\omega)\left(\xi_{n+1}(\omega)-T\left(\omega, \xi_{n+1}(\omega)\right)\right) \\
& -2 \alpha_{n}(\omega) \xi_{n}(\omega)+2\left(\alpha_{n}(\omega)\right)^{2}\left(\xi_{n}(\omega)-T\left(\omega, \eta_{n}(\omega)\right)\right) \\
& +\alpha_{n}(\omega) \xi_{n}(\omega)+\alpha_{n}(\omega)\left(T\left(\omega, \xi_{n+1}(\omega)\right)-T\left(\omega, \eta_{n}(\omega)\right)\right) \\
= & \left(1+\alpha_{n}(\omega)\right) \xi_{n+1}(\omega)+\alpha_{n}(\omega)\left(\xi_{n+1}(\omega)-T\left(\omega, \xi_{n+1}(\omega)\right)\right)+\left(1-2 \alpha_{n}(\omega)\right) \xi_{n}(\omega) \\
& +2\left(\alpha_{n}(\omega)\right)^{2}\left(\xi_{n}(\omega)-T\left(\omega, \eta_{n}(\omega)\right)\right)+\alpha_{n}(\omega)\left(T\left(\omega, \xi_{n+1}(\omega)\right)-T\left(\omega, \eta_{n}(\omega)\right)\right),
\end{aligned}
$$


for each $\omega \in \Omega$. Also,

$$
\begin{aligned}
\zeta_{n}(\omega)= & \zeta_{n+1}(\omega)+\alpha_{n}(\omega) \zeta_{n}(\omega)-\alpha_{n}(\omega) T\left(\omega, \zeta_{n}(\omega)\right) \\
= & \left(1+\alpha_{n}(\omega)\right) \zeta_{n+1}(\omega)+\alpha_{n}(\omega)\left(\zeta_{n+1}(\omega)-T\left(\omega, \zeta_{n+1}(\omega)\right)\right) \\
& -2 \alpha_{n}(\omega) \zeta_{n+1}(\omega)+\alpha_{n}(\omega) \zeta_{n}(\omega)+\alpha_{n}(\omega)\left(T\left(\omega, \zeta_{n+1}(\omega)\right)-T\left(\omega, \zeta_{n}(\omega)\right)\right) \\
= & \left(1+\alpha_{n}(\omega)\right) \zeta_{n+1}(\omega)+\alpha_{n}(\omega)\left(\zeta_{n+1}(\omega)-T\left(\omega, \zeta_{n+1}(\omega)\right)\right) \\
& -2 \alpha_{n}(\omega)\left(\zeta_{n}(\omega)+\alpha_{n}(\omega)\left(T\left(\omega, \zeta_{n}(\omega)\right)-\zeta_{n}(\omega)\right)\right) \\
& +\alpha_{n}(\omega) \zeta_{n}(\omega)+\alpha_{n}(\omega)\left(T\left(\omega, \zeta_{n+1}(\omega)\right)-T\left(\omega, \zeta_{n}(\omega)\right)\right) \\
= & \left(1+\alpha_{n}(\omega)\right) \zeta_{n+1}(\omega)+\alpha_{n}(\omega)\left(\zeta_{n+1}(\omega)-T\left(\omega, \zeta_{n+1}(\omega)\right)\right) \\
& +2\left(\alpha_{n}(\omega)\right)^{2}\left(\zeta_{n}(\omega)-T\left(\omega, \zeta_{n}(\omega)\right)\right)+\left(1-2 \alpha_{n}(\omega)\right) \alpha_{n}(\omega) \zeta_{n}(\omega) \\
& +\alpha_{n}(\omega)\left(T\left(\omega, \zeta_{n+1}(\omega)\right)-T\left(\omega, \zeta_{n}(\omega)\right)\right),
\end{aligned}
$$

for each $\omega \in \Omega$. From (3.16) and (3.17), we have

$$
\begin{aligned}
\xi_{n}(\omega)-\zeta_{n}(\omega)= & \left(1+\alpha_{n}(\omega)\right)\left(\xi_{n+1}(\omega)-\zeta_{n+1}(\omega)\right)+\alpha_{n}(\omega)\left(T\left(\omega, \zeta_{n+1}(\omega)\right)-T\left(\omega, \xi_{n+1}(\omega)\right)\right) \\
& -\alpha_{n}(\omega)\left(\zeta_{n+1}(\omega)-\xi_{n+1}(\omega)\right)+\left(1-2 \alpha_{n}(\omega)\right) \alpha_{n}(\omega)\left(\zeta_{n}(\omega)-\xi_{n}(\omega)\right) \\
& +2\left(\alpha_{n}(\omega)\right)^{2}\left(\xi_{n}(\omega)-\zeta_{n}(\omega)-T\left(\omega, \eta_{n}(\omega)\right)+T\left(\omega, \zeta_{n}(\omega)\right)\right) \\
& +\alpha_{n}(\omega)\left(T\left(\omega, \xi_{n+1}(\omega)\right)-T\left(\omega, \zeta_{n+1}(\omega)\right)-T\left(\omega, \eta_{n}(\omega)\right)+T\left(\omega, \zeta_{n}(\omega)\right)\right),
\end{aligned}
$$

for every $\omega \in \Omega$. Since $T$ is strongly pseudocontractive random operator, so taking the norm of the sum of the first two terms on the right-hand side of (3.18), we have

$$
\begin{aligned}
& \left\|\left(1+\alpha_{n}(\omega)\right)\left(\xi_{n+1}(\omega)-\zeta_{n+1}(\omega)\right)+\alpha_{n}(\omega)\left(T\left(\omega, \zeta_{n+1}(\omega)\right)-T\left(\omega, \xi_{n+1}(\omega)\right)\right)\right\| \\
& \quad \geq\left\|\xi_{n+1}(\omega)-\zeta_{n+1}(\omega)\right\| .
\end{aligned}
$$

From (3.18), we have

$$
\begin{aligned}
\| \xi_{n}(\omega) & -\zeta_{n}(\omega) \| \\
\geq & \left\|\left(1+\alpha_{n}(\omega)\right)\left(\xi_{n+1}(\omega)-\zeta_{n+1}(\omega)\right)+\alpha_{n}(\omega)\left(T\left(\omega, \zeta_{n+1}(\omega)\right)-T\left(\omega, \xi_{n+1}(\omega)\right)\right)\right\| \\
& +\left(1-2 \alpha_{n}(\omega)\right) \alpha_{n}(\omega)\left\|\left(\zeta_{n}(\omega)-\xi_{n}(\omega)\right)\right\| \\
& -2\left(\alpha_{n}(\omega)\right)^{2}\left\|\xi_{n}(\omega)-\zeta_{n}(\omega)-T\left(\omega, \eta_{n}(\omega)\right)+T\left(\omega, \zeta_{n}(\omega)\right)\right\| \\
& +\alpha_{n}(\omega)\left\|T\left(\omega, \xi_{n+1}(\omega)\right)-T\left(\omega, \zeta_{n+1}(\omega)\right)-T\left(\omega, \eta_{n}(\omega)\right)+T\left(\omega, \zeta_{n}(\omega)\right)\right\| \\
& +\alpha_{n}(\omega)\left\|\zeta_{n+1}(\omega)-\xi_{n+1}(\omega)\right\| \\
\geq & +\alpha_{n}(\omega)\left\|\xi_{n+1}(\omega)-\zeta_{n+1}(\omega)\right\|+\left(1-2 \alpha_{n}(\omega)\right) \alpha_{n}(\omega)\left\|\zeta_{n}(\omega)-\zeta_{n}(\omega)\right\| \\
& -2\left(\alpha_{n}(\omega)\right)^{2}\left\|\xi_{n}(\omega)-\zeta_{n}(\omega)-T\left(\omega, \eta_{n}(\omega)\right)+T\left(\omega, \zeta_{n}(\omega)\right)\right\| \\
& -\alpha_{n}(\omega)\left\|T\left(\omega, \xi_{n+1}(\omega)\right)-T\left(\omega, \zeta_{n+1}(\omega)\right)-T\left(\omega, \eta_{n}(\omega)\right)+T\left(\omega, \zeta_{n}(\omega)\right)\right\|
\end{aligned}
$$


for each $\omega \in \Omega$. Thus we have

$$
\begin{aligned}
1+\alpha_{n}(\omega)\left\|\xi_{n+1}(\omega)-\zeta_{n+1}(\omega)\right\| \leq & \left(1-\left(1-2 \alpha_{n}(\omega)\right) \alpha_{n}(\omega)\right)\left\|\zeta_{n}(\omega)-\xi_{n}(\omega)\right\| \\
& +2\left(\alpha_{n}(\omega)\right)^{2}\left\|\zeta_{n}(\omega)-T\left(\omega, \zeta_{n}(\omega)\right)\right\| \\
& +2\left(\alpha_{n}(\omega)\right)^{2}\left\|\xi_{n}(\omega)-T\left(\omega, \eta_{n}(\omega)\right)\right\| \\
& +\alpha_{n}(\omega)\left\|T\left(\omega, \xi_{n+1}(\omega)\right)-T\left(\omega, \eta_{n}(\omega)\right)\right\| \\
& +\alpha_{n}(\omega)\left\|T\left(\omega, \zeta_{n+1}(\omega)\right)-T\left(\omega, \zeta_{n}(\omega)\right)\right\| .
\end{aligned}
$$

Also,

$$
\begin{aligned}
\| \xi_{n}(\omega) & -T\left(\omega, \eta_{n}(\omega)\right) \| \\
& \leq\left\|\xi_{n}(\omega)-\zeta_{n}(\omega)\right\|+\left\|\zeta_{n}(\omega)-T\left(\omega, \zeta_{n}(\omega)\right)\right\|+\left\|T\left(\omega, \zeta_{n}(\omega)\right)-T\left(\omega, \eta_{n}(\omega)\right)\right\| \\
& \leq\left\|\xi_{n}(\omega)-\zeta_{n}(\omega)\right\|+\left\|\zeta_{n}(\omega)-T\left(\omega, \zeta_{n}(\omega)\right)\right\|+L\left\|\zeta_{n}(\omega)-\eta_{n}(\omega)\right\|,
\end{aligned}
$$

for each $\omega \in \Omega$. Consider

$$
\begin{aligned}
\left\|\zeta_{n}(\omega)-\eta_{n}(\omega)\right\|= & \left\|\left(1-\beta_{n}(\omega)\right)\left(\zeta_{n}(\omega)-\xi_{n}(\omega)\right)+\beta_{n}(\omega)\left(\zeta_{n}(\omega)-T\left(\omega, \xi_{n}(\omega)\right)\right)\right\| \\
\leq & \left(1-\beta_{n}(\omega)\right)\left\|\zeta_{n}(\omega)-\xi_{n}(\omega)\right\|+\beta_{n}(\omega)\left\|\zeta_{n}(\omega)-T\left(\omega, \xi_{n}(\omega)\right)\right\| \\
\leq & \left(1-\beta_{n}(\omega)\right)\left\|\zeta_{n}(\omega)-\xi_{n}(\omega)\right\|+\beta_{n}(\omega)\left\|T\left(\omega, \zeta_{n}(\omega)\right)-T\left(\omega, \xi_{n}(\omega)\right)\right\| \\
& +\beta_{n}(\omega)\left\|\zeta_{n}(\omega)-T\left(\omega, \zeta_{n}(\omega)\right)\right\| \\
\leq & \left(1-\beta_{n}(\omega)\right)\left\|\zeta_{n}(\omega)-\xi_{n}(\omega)\right\|+\beta_{n}(\omega) L\left\|\zeta_{n}(\omega)-\xi_{n}(\omega)\right\| \\
& +\beta_{n}(\omega)\left\|\zeta_{n}(\omega)-T\left(\omega, \zeta_{n}(\omega)\right)\right\| \\
= & \left(1-\beta_{n}(\omega)+\beta_{n}(\omega) L\right)\left\|\zeta_{n}(\omega)-\xi_{n}(\omega)\right\|+\beta_{n}(\omega)\left\|\zeta_{n}(\omega)-T\left(\omega, \zeta_{n}(\omega)\right)\right\| \\
\leq & L\left\|\zeta_{n}(\omega)-\xi_{n}(\omega)\right\|+\beta_{n}(\omega)\left\|\zeta_{n}(\omega)-T\left(\omega, \zeta_{n}(\omega)\right)\right\|,
\end{aligned}
$$

for each $\omega \in \Omega$. Now,

$$
\begin{aligned}
\left\|\xi_{n}(\omega)-T\left(\omega, \eta_{n}(\omega)\right)\right\| \leq & \left\|\xi_{n}(\omega)-\zeta_{n}(\omega)\right\|+\left\|\zeta_{n}(\omega)-T\left(\omega, \zeta_{n}(\omega)\right)\right\| \\
& +L\left(L\left\|\zeta_{n}(\omega)-\xi_{n}(\omega)\right\|+\beta_{n}(\omega)\left\|\zeta_{n}(\omega)-T\left(\omega, \zeta_{n}(\omega)\right)\right\|\right) \\
\leq & \left(1+L^{2}\right)\left\|\xi_{n}(\omega)-\zeta_{n}(\omega)\right\|+\left(1+\beta_{n}(\omega) L\right)\left\|\zeta_{n}(\omega)-T\left(\omega, \zeta_{n}(\omega)\right)\right\|,
\end{aligned}
$$

for each $\omega \in \Omega$. Consider

$$
\begin{aligned}
\left\|T\left(\omega, \xi_{n+1}(\omega)\right)-T\left(\omega, \eta_{n}(\omega)\right)\right\| \leq & L\left\|\xi_{n+1}(\omega)-\eta_{n}(\omega)\right\| \\
= & L\left\|\left(1-\alpha_{n}(\omega)\right) \xi_{n}(\omega)+\alpha_{n}(\omega) T\left(\omega, \eta_{n}(\omega)\right)-\eta_{n}(\omega)\right\| \\
\leq & L\left[\left(1-\alpha_{n}(\omega)\right)\left\|\xi_{n}(\omega)-\eta_{n}(\omega)\right\|\right. \\
& \left.+\alpha_{n}(\omega)\left\|T\left(\omega, \eta_{n}(\omega)\right)-\eta_{n}(\omega)\right\|\right],
\end{aligned}
$$


12 Equivalence of random iterative procedure

for each $\omega \in \Omega$. Also,

$$
\begin{aligned}
\| T(\omega, & \left.\eta_{n}(\omega)\right)-\eta_{n}(\omega) \| \\
\leq & \left\|T\left(\omega, \eta_{n}(\omega)\right)-T\left(\omega, \zeta_{n}(\omega)\right)\right\|+\left\|T\left(\omega, \zeta_{n}(\omega)\right)-\zeta_{n}(\omega)\right\|+\left\|\eta_{n}(\omega)-\zeta_{n}(\omega)\right\| \\
\leq & L\left\|\eta_{n}(\omega)-\zeta_{n}(\omega)\right\|+\left\|T\left(\omega, \zeta_{n}(\omega)\right)-\zeta_{n}(\omega)\right\|+\left\|\eta_{n}(\omega)-\zeta_{n}(\omega)\right\| \\
\leq & (1+L)\left\|\eta_{n}(\omega)-\zeta_{n}(\omega)\right\|+\left\|T\left(\omega, \zeta_{n}(\omega)\right)-\zeta_{n}(\omega)\right\| \\
\leq & (1+L)\left[L\left\|\zeta_{n}(\omega)-\xi_{n}(\omega)\right\|+\beta_{n}(\omega)\left\|\zeta_{n}(\omega)-T\left(\omega, \zeta_{n}(\omega)\right)\right\|\right] \\
& +\left\|T\left(\omega, \zeta_{n}(\omega)\right)-\zeta_{n}(\omega)\right\| \\
= & (1+L) L\left\|\zeta_{n}(\omega)-\zeta_{n}(\omega)\right\|+\left[(1+L) \beta_{n}(\omega)+1\right]\left\|\zeta_{n}(\omega)-T\left(\omega, \zeta_{n}(\omega)\right)\right\|,
\end{aligned}
$$

for every $\omega \in \Omega$. Hence we reach the following inequality:

$$
\begin{aligned}
\left\|\xi_{n}(\omega)-\eta_{n}(\omega)\right\|= & \left\|\xi_{n}(\omega)-\left(1-\beta_{n}(\omega)\right) \xi_{n}(\omega)+\beta_{n}(\omega) T\left(\omega, \xi_{n}(\omega)\right)\right\| \\
= & \beta_{n}(\omega)\left\|\xi_{n}(\omega)-T\left(\omega, \xi_{n}(\omega)\right)\right\| \\
\leq & \beta_{n}(\omega)\left(\left\|\xi_{n}(\omega)-\zeta_{n}(\omega)\right\|+\left\|T\left(\omega, \zeta_{n}(\omega)\right)-\zeta_{n}(\omega)\right\|\right. \\
& \left.\quad+\left\|T\left(\omega, \zeta_{n}(\omega)\right)-T\left(\omega, \xi_{n}(\omega)\right)\right\|\right) \\
\leq & \beta_{n}(\omega)\left[(1+L)\left\|\xi_{n}(\omega)-\zeta_{n}(\omega)\right\|+\left\|T\left(\omega, \zeta_{n}(\omega)\right)-\zeta_{n}(\omega)\right\|\right],
\end{aligned}
$$

for each $\omega \in \Omega$. We have

$$
\begin{aligned}
& \left\|T\left(\omega, \xi_{n+1}(\omega)\right)-T\left(\omega, \eta_{n}(\omega)\right)\right\| \\
& \leq L\left[( 1 - \alpha _ { n } ( \omega ) ) \left[\beta_{n}(\omega)\left[(1+L)\left\|\xi_{n}(\omega)-\zeta_{n}(\omega)\right\|+\left\|T\left(\omega, \zeta_{n}(\omega)\right)-\zeta_{n}(\omega)\right\|\right]\right.\right. \\
& \quad+\alpha_{n}(\omega)(1+L)\left[L\left\|\zeta_{n}(\omega)-\xi_{n}(\omega)\right\|+\beta_{n}(\omega)\left\|\zeta_{n}(\omega)-T\left(\omega, \zeta_{n}(\omega)\right)\right\|\right] \\
& \left.\quad+\alpha_{n}(\omega)\left\|\zeta_{n}(\omega)-T\left(\omega, \zeta_{n}(\omega)\right)\right\|\right] \\
& \leq L\left(1-\alpha_{n}(\omega)\right) \beta_{n}(\omega)(1+L)+\alpha_{n}(\omega)(1+L) L^{2}\left\|\xi_{n}(\omega)-\zeta_{n}(\omega)\right\| \\
& \quad+L\left(1-\alpha_{n}(\omega)\right) \beta_{n}(\omega)+\alpha_{n}(\omega) L(1+L) \beta_{n}(\omega)+1\left\|T\left(\omega, \zeta_{n}(\omega)\right)-\zeta_{n}(\omega)\right\|,
\end{aligned}
$$

for every $\omega \in \Omega$. Consider

$$
\begin{aligned}
\| \xi_{n}(\omega) & -T\left(\omega, \eta_{n}(\omega)\right) \| \\
\leq & \left\|\xi_{n}(\omega)-\zeta_{n}(\omega)\right\|+\left\|\zeta_{n}(\omega)-T\left(\omega, \zeta_{n}(\omega)\right)\right\|+\left\|T\left(\omega, \eta_{n}(\omega)\right)-T\left(\omega, \zeta_{n}(\omega)\right)\right\| \\
\leq & \left\|\xi_{n}(\omega)-\zeta_{n}(\omega)\right\|+\left\|\zeta_{n}(\omega)-T\left(\omega, \zeta_{n}(\omega)\right)\right\|+L\left\|\eta_{n}(\omega)-\zeta_{n}(\omega)\right\| \\
\leq & \left\|\xi_{n}(\omega)-\zeta_{n}(\omega)\right\|+\left\|\zeta_{n}(\omega)-T\left(\omega, \zeta_{n}(\omega)\right)\right\| \\
& +L\left(L\left\|\zeta_{n}(\omega)-\xi_{n}(\omega)\right\|+\beta_{n}(\omega)\left\|\zeta_{n}(\omega)-T\left(\omega, \zeta_{n}(\omega)\right)\right\|\right) \\
= & \left(1+L^{2}\right)\left\|\xi_{n}(\omega)-\zeta_{n}(\omega)\right\|+\left(1+L \beta_{n}(\omega)\right)\left\|\zeta_{n}(\omega)-T\left(\omega, \zeta_{n}(\omega)\right)\right\|,
\end{aligned}
$$


for each $\omega \in \Omega$. Finally we arrive at

$$
\begin{aligned}
& 1+\left(\alpha_{n}(\omega)\right)^{2}\left\|\xi_{n+1}(\omega)-\zeta_{n+1}(\omega)\right\| \\
& \leq\left(1-\left(1-2 \alpha_{n}(\omega)\right) \alpha_{n}(\omega)\right)\left\|\zeta_{n}(\omega)-\xi_{n}(\omega)\right\| \\
&+2\left(\alpha_{n}(\omega)\right)^{2}\left(1+L^{2}\left\|\zeta_{n}(\omega)-\xi_{n}(\omega)\right\|+1+\beta_{n}(\omega) L\left\|\zeta_{n}(\omega)-T\left(\omega, \zeta_{n}(\omega)\right)\right\|\right) \\
&+2\left(\alpha_{n}(\omega)\right)^{2}\left\|T\left(\omega, \zeta_{n}(\omega)\right)-\zeta_{n}(\omega)\right\| \\
&+\alpha_{n}(\omega)\left\|T\left(\omega, \zeta_{n+1}(\omega)\right)-T\left(\omega, \zeta_{n}(\omega)\right)\right\| \\
&+\alpha_{n}(\omega)\left(L\left(1-\alpha_{n}(\omega)\right) \beta_{n}(\omega)(1+L)\right. \\
&\left.+\alpha_{n}(\omega)(1+L) L^{2}\right)\left\|\xi_{n}(\omega)-\zeta_{n}(\omega)\right\| \\
&+\alpha_{n}(\omega)\left(L\left(1-\alpha_{n}(\omega)\right) \beta_{n}(\omega)+\alpha_{n}(\omega) L(1+L) \beta_{n}(\omega)+1\right)\left\|T\left(\omega, \zeta_{n}(\omega)\right)-\zeta_{n}(\omega)\right\| .
\end{aligned}
$$

So for every $\omega \in \Omega$,

$$
\begin{aligned}
\| \xi_{n+1}(\omega) & -\zeta_{n+1}(\omega) \| \\
\leq & \left(1-\left(1-2 \alpha_{n}(\omega)\right) \alpha_{n}(\omega)\right)+2\left(\alpha_{n}(\omega)\right)^{2}\left(1+L^{2}\right) \\
& +\alpha_{n}(\omega)\left(L\left(1-\alpha_{n}(\omega)\right) \beta_{n}(\omega)(1+L)+\alpha_{n}(\omega)(1+L) L^{2}\right)\left\|\xi_{n}(\omega)-\zeta_{n}(\omega)\right\| \\
& +2\left(\alpha_{n}(\omega)\right)^{2}\left(2+\beta_{n}(\omega) L\right) \\
& \times\left[\left(1+L^{2}\right)+\alpha_{n}(\omega)\left\{L\left(1-\alpha_{n}(\omega)\right) \beta_{n}(\omega)+\alpha_{n}(\omega) L(1+L) \beta_{n}(\omega)\right.\right. \\
& \left.\left.\quad+1\left\|T\left(\omega, \zeta_{n}(\omega)\right)-\zeta_{n}(\omega)\right\|\right\}\right] \\
& +\alpha_{n}(\omega)\left\|T\left(\omega, \zeta_{n+1}(\omega)\right)-T\left(\omega, \zeta_{n}(\omega)\right)\right\| .
\end{aligned}
$$

We may write it as

$$
\varphi_{n+1}(\omega) \leq \delta_{n}(\omega) \varphi_{n}(\omega)+\sigma_{n}(\omega),
$$

where, for each $\omega \in \Omega$,

$$
\begin{aligned}
\varphi_{n}(\omega)= & \left\|\left(\xi_{n}(\omega)-\zeta_{n}(\omega)\right)\right\|, \\
\delta_{n}(\omega)= & \left(1-\left(1-2 \alpha_{n}(\omega)\right) \alpha_{n}(\omega)\right)+2 \alpha_{n}^{2}\left(1+L^{2}\right) \\
& +\alpha_{n}(\omega)\left(L\left(1-\alpha_{n}(\omega)\right) \beta_{n}(\omega)(1+L)+\alpha_{n}(\omega)(1+L) L^{2}\right), \\
\sigma_{n}(\omega)=\alpha_{n}(\omega)[ & 2 \alpha_{n}(\omega)\left(2+\beta_{n}(\omega) L\right) \\
& \times\left(\left(1+L^{2}\right)+\left(L\left(1-\alpha_{n}(\omega)\right) \beta_{n}(\omega)+\alpha_{n}(\omega) L(1+L) \beta_{n}(\omega)+1\right)\right) \\
& \left.\times\left\|T\left(\omega, \zeta_{n}(\omega)\right)-\zeta_{n}(\omega)\right\|+\left\|T\left(\omega, \zeta_{n+1}(\omega)\right)-T\left(\omega, \zeta_{n}(\omega)\right)\right\|\right] .
\end{aligned}
$$


14 Equivalence of random iterative procedure

Consider

$$
\begin{aligned}
L\left(1-\alpha_{n}(\omega)\right) \beta_{n}(\omega)(1+L)+\alpha_{n}(\omega)(1+L) L^{2} & \leq L(1+L)\left[\left(1-\alpha_{n}(\omega)\right) \beta_{n}(\omega)+\alpha_{n}(\omega) L\right] \\
& \leq L(1+L)\left[L \beta_{n}(\omega)+\alpha_{n}(\omega) L\right] \\
& \leq L^{2}(1+L)\left(\alpha_{n}(\omega)+\beta_{n}(\omega)\right),
\end{aligned}
$$

for each $\omega \in \Omega$. Since $\lim _{n \rightarrow \infty} \alpha_{n}(\omega)=0=\lim _{n \rightarrow \infty} \beta_{n}(\omega)$ and $\sum_{n=1}^{\infty} \alpha_{n}(\omega)=\infty$, so

$$
\alpha_{n}(\omega) \leq \frac{1}{50\left(1+L^{2}\right)}, \quad \alpha_{n}(\omega)+\beta_{n}(\omega) \leq \frac{1}{5(1+L) L^{2}}
$$

for every $\omega \in \Omega$. Thus,

$$
\begin{aligned}
\delta_{n}(\omega) \leq & \left(1-\left(1-2 \alpha_{n}(\omega)\right) \alpha_{n}(\omega)\right)+2\left(\alpha_{n}(\omega)\right)^{2}\left(1+L^{2}\right) \\
& +\alpha_{n}(\omega) L^{2}(1+L)\left(\alpha_{n}(\omega)+\beta_{n}(\omega)\right) \\
\leq & \left(1-\left(1-2 \alpha_{n}(\omega)\right) \alpha_{n}(\omega)\right)+\frac{1}{25} \alpha_{n}(\omega)+\frac{1}{5} \alpha_{n}(\omega) \\
\leq & \left(1-\left(1-2 \alpha_{n}(\omega)\right) \alpha_{n}(\omega)\right)+\frac{2}{5} \alpha_{n}(\omega) \\
\leq & 1-\frac{4}{5} \alpha_{n}(\omega)+\frac{2}{5} \alpha_{n}(\omega)=1-\frac{2}{5} \alpha_{n}(\omega), \quad \text { for sufficiently large } n .
\end{aligned}
$$

So,

$$
\begin{gathered}
\varphi_{n+1}(\omega) \leq \delta_{n}(\omega) \varphi_{n}(\omega)+\sigma_{n}(\omega) \\
\varphi_{n+1}(\omega) \leq\left(1-\frac{2}{5} \alpha_{n}(\omega)\right) \varphi_{n}(\omega)+\sigma_{n}(\omega),
\end{gathered}
$$

for each $\omega \in \Omega$. The application of Lemma 2.10 gives

$$
\lim _{n \rightarrow \infty}\left\|\xi_{n}(\omega)-\zeta_{n}(\omega)\right\|=0, \quad \text { for each } \omega \in \Omega
$$

Now, since $\lim _{n \rightarrow \infty}\left\|\zeta_{n}(\omega)-\xi^{*}(\omega)\right\|=0$, for each $\omega \in \Omega$, consider

$$
\left\|\xi_{n}(\omega)-\xi^{*}(\omega)\right\| \leq\left\|\xi_{n}(\omega)-\zeta_{n}(\omega)\right\|+\left\|\zeta_{n}(\omega)-\xi^{*}(\omega)\right\| \longrightarrow 0,
$$

as $n \rightarrow \infty$, for each $\omega \in \Omega$. Hence we have $\lim _{n \rightarrow \infty}\left\|\xi_{n}(\omega)-\xi^{*}(\omega)\right\|=0$, for each $\omega \in$ $\Omega$.

Corollary 3.4. Let $F$ be a nonempty closed bounded and convex subset of a separable reflexive Banach space $X$ with $0 \in \operatorname{int}(F)$. Let $T: \Omega \times F \rightarrow F$ be a $k(\omega)$-contraction random operator satisfying the Leray-Schauder boundary condition for $z=0$. Let $\xi_{0}=\zeta_{0}$ be a fixed measurable mapping from $\Omega$ to $F$. Define the sequences of functions $\left\{\eta_{n}\right\}$ and $\left\{\xi_{n}\right\}$ as 
given below:

$$
\begin{gathered}
\eta_{n}(\omega)=\beta_{n}(\omega) T\left(\omega, \xi_{n}(\omega)\right)+\left(1-\beta_{n}(\omega)\right) \xi_{n}(\omega), \\
\xi_{n+1}(\omega)=\alpha_{n}(\omega) T\left(\omega, \eta_{n}(\omega)\right)+\left(1-\alpha_{n}(\omega)\right) \xi_{n}(\omega), \quad \text { for each } \omega \in \Omega, \\
\zeta_{n+1}(\omega)=\left(1-\alpha_{n}(\omega)\right) \zeta_{n}(\omega)+\alpha_{n}(\omega) T\left(\omega, \zeta_{n}(\omega)\right), \quad \text { for each } \omega \in \Omega,
\end{gathered}
$$

$n=0,1,2, \ldots$, where $\alpha_{n}$ and $\beta_{n}: \Omega \rightarrow[0,1]$ are measurable mappings for each $n \in \mathbb{N}$, and $\lim _{n \rightarrow \infty} \alpha_{n}(\omega)=0=\lim _{n \rightarrow \infty} \beta_{n}(\omega)$ and $\sum_{n \geq 0} \alpha_{n}(\omega)=\infty$, for each $\omega \in \Omega$. Then the sequence of measurable mappings $\left\{\xi_{n}\right\}$ converges strongly to the random fixed point $\xi^{*}$ of $T$ if and only if the sequence of measurable mappings $\left\{\zeta_{n}\right\}$ converges strongly to the random fixed point $\xi^{*}$ of $T$.

THeORem 3.5. Let $F$ be a nonempty closed bounded and convex subset of a separable Hilbert space $H$ and let $T_{i}: \Omega \times F \rightarrow F$ be a completely continuous and nonexpansive random operator for each $i \in J=\{1,2, \ldots, \mathbb{N}\}$. Let $\xi_{0}: \Omega \rightarrow F$ be any fixed measurable mapping. The sequence $\left\{\xi_{n}\right\}$ of measurable mappings from $\Omega$ to $F$ defined by random implicit iterative procedure (2.11) converges weakly to common random fixed point of the random operators $\left\{T_{i}: i \in J\right\}$.

Proof. For any $\xi \in D$, using the Hilbert space identity, we have

$$
\begin{aligned}
\left\|\xi_{n}(\omega)-\xi(\omega)\right\|^{2}= & \left\|\alpha_{n}(\omega) \xi_{n-1}(\omega)-\left(1-\alpha_{n}(\omega)\right) T_{n}\left(\omega, \xi_{n}(\omega)\right)-\xi(\omega)\right\|^{2} \\
= & \alpha_{n}(\omega)\left\|\xi_{n-1}(\omega)-\xi(\omega)\right\|^{2}+\left(1-\alpha_{n}(\omega)\right)\left\|T_{n}\left(\omega, \xi_{n}(\omega)\right)-\xi(\omega)\right\|^{2} \\
& -\alpha_{n}(\omega)\left(1-\alpha_{n}(\omega)\right)\left\|\xi_{n-1}(\omega)-T_{n}\left(\omega, \xi_{n}(\omega)\right)\right\|^{2},
\end{aligned}
$$

for every $\omega \in \Omega$. Since $T_{n}$ is nonexpansive random operator for each $n \in J$, so

$$
\begin{aligned}
\left\|\xi_{n}(\omega)-\xi(\omega)\right\|^{2} \leq & \alpha_{n}(\omega)\left\|\xi_{n-1}(\omega)-\xi(\omega)\right\|^{2}+\left(1-\alpha_{n}(\omega)\right)\left\|\xi_{n}(\omega)-\xi(\omega)\right\|^{2} \\
& -\alpha_{n}(\omega)\left(1-\alpha_{n}(\omega)\right)\left\|\xi_{n-1}(\omega)-T_{n}\left(\omega, \xi_{n}(\omega)\right)\right\|^{2}
\end{aligned}
$$

for each $\omega \in \Omega$. Hence,

$$
\left\|\xi_{n}(\omega)-\xi(\omega)\right\|^{2} \leq\left\|\xi_{n-1}(\omega)-\xi(\omega)\right\|^{2}
$$

for each $\omega \in \Omega$. We also have

$$
\sum_{n=1}^{\infty}\left\|\xi_{n-1}(\omega)-T_{n}\left(\omega, \xi_{n}(\omega)\right)\right\|^{2}<\infty
$$

which yields

$$
\lim _{n \rightarrow \infty}\left\|\xi_{n-1}(\omega)-T_{n}\left(\omega, \xi_{n}(\omega)\right)\right\|=0,
$$

for every $\omega \in \Omega$. Now,

$$
\left\|\xi_{n}(\omega)-\xi_{n-1}(\omega)\right\|=\left(1-\alpha_{n}(\omega)\right)\left\|\xi_{n-1}(\omega)-T_{n}\left(\omega, \xi_{n}(\omega)\right)\right\| \longrightarrow 0, \quad \text { as } n \longrightarrow \infty,
$$


for each $\omega \in \Omega$. For each $i \in J$, consider

$$
\begin{aligned}
\left\|\xi_{n}(\omega)-T_{n+i}\left(\omega, \xi_{n}(\omega)\right)\right\| \leq & \left\|\xi_{n}(\omega)-\xi_{n+i}(\omega)\right\|+\left\|\xi_{n+i}(\omega)-T_{n+i}\left(\omega, \xi_{n+i}(\omega)\right)\right\| \\
& +\left\|T_{n+i}\left(\omega, \xi_{n+i}(\omega)\right)-T_{n+i}\left(\omega, \xi_{n}(\omega)\right)\right\| \\
\leq & 2\left\|\xi_{n}(\omega)-\xi_{n+i}(\omega)\right\|+\left\|\xi_{n+i}(\omega)-T_{n+i}\left(\omega, \xi_{n+i}(\omega)\right)\right\|,
\end{aligned}
$$

for each $\omega \in \Omega$. So for every $i \in J$, we have

$$
\lim _{n \rightarrow \infty}\left\|\xi_{n}(\omega)-T_{n+i}\left(\omega, \xi_{n}(\omega)\right)\right\|=0, \quad \text { for each } \omega \in \Omega .
$$

Assume that $\xi^{*}(\omega)$ is a weak cluster point of $\left\{\xi_{n}(\omega)\right\}$ and $w-\lim _{k} \xi_{n_{k}}(\omega)=\xi^{*}(\omega)$, for every $\omega \in \Omega$. Assuming $n_{k} \equiv j \bmod \mathbb{N}$ for some $j \in J$ and for all $k$, for any fixed $l \in J$, we can find $i \in J$, independent of $k$, such that $n_{k}+i \equiv l \bmod \mathbb{N}$ for all $k$. From (3.48), we have

$$
\lim _{n \rightarrow \infty}\left\|\xi_{n_{k}}(\omega)-T_{l}\left(\omega, \xi_{n_{k}}(\omega)\right)\right\|=0, \quad \text { for every } \omega \in \Omega
$$

Since for each $\omega \in \Omega, I-T(\omega, \cdot)$ is demiclosed at 0 , therefore it follows that $T_{l}(\omega$, $\left.\xi^{*}(\omega)\right)=\xi^{*}(\omega)$, for each $\omega \in \Omega$. The measurability of $\xi^{*}$ follows from the fact that $T$ is completely continuous random operator and from Remark 2.3. We reach the conclusion that $\xi^{*}$ is the random fixed point of $T_{l}$. Since $l$ is arbitrary, we get $\xi^{*} \in D$. Now we show that the entire sequence $\left\{\xi_{n}\right\}$ converges weakly to $\xi^{*}$. Assume that $\eta(\omega)$ is another weak cluster point of $\left\{\xi_{n}(\omega)\right\}$ and $w-\lim _{j} \xi_{m_{j}}(\omega)=\eta(\omega)$, for every $\omega \in \Omega$. Using the similar argument as above, $\eta \in D$. Using (3.43) for every $\xi \in D$, we have $\lim _{n}\left\|\xi_{n}(\omega)-\xi(\omega)\right\|$, for each $\omega \in \Omega$. Now we prove $\xi^{*}(\omega)=\eta(\omega)$, for each $\omega$ in $\Omega$. If it is not so, then for some $\omega \in \Omega, \xi^{*}(\omega) \neq \eta(\omega)$; by Opial's condition,

$$
\begin{aligned}
\lim _{n}\left\|\xi_{n}(\omega)-\xi^{*}(\omega)\right\| \\
\quad=\lim _{k}\left\|\xi_{n_{k}}(\omega)-\xi^{*}(\omega)\right\|<\lim _{n}\left\|\xi_{m_{j}}(\omega)-\eta(\omega)\right\| \\
=\lim _{j}\left\|\xi_{m_{j}}(\omega)-\eta(\omega)\right\|<\lim _{j}\left\|\xi_{m_{j}}(\omega)-\xi^{*}(\omega)\right\| \\
=\lim _{n}\left\|\xi_{n}(\omega)-\xi^{*}(\omega)\right\| .
\end{aligned}
$$

This contradiction concludes the proof.

\section{Stability of random iterative procedure involving weakly random operator}

Theorem 4.1. Let $F$ be a nonempty closed bounded and convex subset of a separable complete metric space $X$ and let $T: \Omega \times F \rightarrow F$ be a weakly contractive random operator. Let $\xi_{0}: \Omega \rightarrow F$ be any fixed measurable mapping. Then the random Picard iterative procedure $\left\{T, \xi_{n+1}(\omega)\right\}$ for $n=0,1,2, \ldots$, generating the sequence $\left\{\xi_{n+1}\right\}$ of measurable mappings from $\Omega$ to $F$, is stable with respect to random operator $T$.

Proof. The existence of a unique random fixed point of weakly contractive random operator and convergence of random Picard iterative procedure to the random fixed point of weakly contractive random operator $T$ can be shown. Let $\xi^{*} \in \operatorname{RF}(T)$. Let $\left\{\eta_{n}\right\}$ be the sequence of measurable mappings from $\Omega$ to $F$. We will show that $\lim _{n \rightarrow \infty} k_{n}(\omega)=0$ if and 
only if $\lim _{n \rightarrow \infty} d\left(\eta_{n}(\omega), \xi^{*}(\omega)\right)=0$, for each $\omega \in \Omega$. Let, for every $\omega \in \Omega, \lim _{n \rightarrow \infty} d\left(\eta_{n}(\omega)\right.$, $\left.\xi^{*}(\omega)\right)=0$. Now employing the continuity of $T$, we have $\lim _{n \rightarrow \infty} k_{n}(\omega)=\lim _{n \rightarrow \infty} d\left(\eta_{n+1}(\omega)\right.$, $\left.T\left(\omega, \eta_{n}(\omega)\right)\right)=d\left(\xi^{*}(\omega), T\left(\omega, \xi^{*}(\omega)\right)\right)=0$, for each $\omega \in \Omega$. Suppose that $\lim _{n \rightarrow \infty} k_{n}(\omega)=$ 0 , for each $\omega \in \Omega$. Consider

$$
\begin{aligned}
d\left(\xi^{*}(\omega), \eta_{n+1}(\omega)\right) \leq & d\left(\xi^{*}(\omega), \xi_{n+1}(\omega)\right)+d\left(\xi_{n+1}(\omega), T\left(\omega, \eta_{n}(\omega)\right)\right) \\
& +d\left(T\left(\omega, \eta_{n}(\omega)\right), \eta_{n+1}(\omega)\right) \\
= & d\left(\xi^{*}(\omega), \xi_{n+1}(\omega)\right)+d\left(T\left(\omega, \xi_{n}(\omega)\right), T\left(\omega, \eta_{n}(\omega)\right)\right)+k_{n}(\omega) \\
\leq & d\left(\xi^{*}(\omega), \xi_{n+1}(\omega)\right)+d\left(\xi_{n}(\omega), \eta_{n}(\omega)\right)-\Psi\left(d\left(\xi_{n}(\omega), \eta_{n}(\omega)\right)\right)+k_{n}(\omega),
\end{aligned}
$$

for each $\omega \in \Omega$. Now,

$$
\begin{aligned}
d\left(\eta_{n+1}(\omega), \xi_{n+1}(\omega)\right) & =d\left(T\left(\omega, \xi_{n}(\omega)\right), T\left(\omega, \eta_{n}(\omega)\right)\right) \\
& \leq d\left(\xi_{n}(\omega), \eta_{n}(\omega)\right)-\Psi\left(d\left(\xi_{n}(\omega), \eta_{n}(\omega)\right)\right) \leq d\left(\xi_{n}(\omega), \eta_{n}(\omega)\right),
\end{aligned}
$$

for every $\omega \in \Omega$. Since $\Psi$ is positive on $(0, \infty)$, this implies that $\left\{d\left(\xi_{n}(\omega), \eta_{n}(\omega)\right)\right\}$ is a nonnegative and nonincreasing sequence of numbers, for every $\omega \in \Omega$. Hence it converges to $L$ such that $L \geq 0$. Now if $L>0$, we have

$$
\Psi\left(d\left(\xi_{n}(\omega), \eta_{n}(\omega)\right)\right) \geq \Psi(L)>0,
$$

for each $\omega \in \Omega$. Thus,

$$
\begin{aligned}
d\left(\xi_{n+1}(\omega), \eta_{n+1}(\omega)\right) & =d\left(T\left(\omega, \xi_{n}(\omega)\right), T\left(\omega, \eta_{n}(\omega)\right)\right) \\
& \leq d\left(\xi_{n}(\omega), \eta_{n}(\omega)\right)-\Psi(L), \quad \text { for every } \omega \in \Omega .
\end{aligned}
$$

Continuing this process, we reach the stage

$$
\begin{aligned}
d\left(\xi_{m+\mathbb{N}}(\omega), \eta_{m+\mathbb{N}}(\omega)\right) & =d\left(T\left(\omega, \xi_{m+\mathbb{N}-1}(\omega)\right), T\left(\omega, \eta_{m+\mathbb{N}-1}(\omega)\right)\right) \\
& \leq d\left(\xi_{m}(\omega), \eta_{m}(\omega)\right)-\mathbb{N} \Psi(L),
\end{aligned}
$$

for every $\omega \in \Omega$. Therefore,

$$
d\left(\xi_{m+\mathbb{N}}(\omega), \eta_{m+\mathbb{N}}(\omega)\right)+\mathbb{N} \Psi(L) \leq d\left(\xi_{m}(\omega), \eta_{m}(\omega)\right)
$$

This gives a contradiction for $\mathbb{N}$ large enough. Thus $L=0$. Next, we have

$$
\begin{aligned}
d\left(\eta_{n+1}(\omega), \xi^{*}(\omega)\right) \leq & d\left(\eta_{n+1}(\omega), T\left(\omega, \eta_{n}(\omega)\right)\right)+d\left(T\left(\omega, \eta_{n}(\omega)\right), \xi_{n+1}(\omega)\right) \\
& +d\left(\xi_{n+1}(\omega), \xi^{*}(\omega)\right)
\end{aligned}
$$

for each $\omega \in \Omega$. Since $\lim _{n \rightarrow \infty} k_{n}(\omega)=0$ for every $\omega \in \Omega$, the result follows.

Corollary 4.2. Let $X$ be a separable complete metric space and let $T: \Omega \times X \rightarrow X$ be a weakly contractive random operator. Let $\xi_{0}: \Omega \rightarrow X$ be any fixed measurable mapping. Then the random Kirk iterative procedure $\left\{T, \xi_{n+1}(\omega)\right\}$ for $n=0,1,2, \ldots$, generating the sequence $\left\{\xi_{n+1}\right\}$ of measurable mappings from $\Omega$ to $X$, is stable with respect to random operator $T$. 
Using similar arguments as in Theorem 3.1, we have the following random stability theorem.

Theorem 4.3. Let $X$ be a separable complete metric space and let $T: \Omega \times X \rightarrow X$ be a $k(\omega)$ contraction random operator, where $k: \Omega \rightarrow(0,1)$ is a measurable mapping. Let $\xi_{0}: \Omega \rightarrow X$ be any fixed measurable mapping. Then the random Picard iterative procedure $\left\{T, \xi_{n+1}(\omega)\right\}$ for $n=0,1,2, \ldots$, generating the sequence $\left\{\xi_{n+1}\right\}$ of measurable mappings from $\Omega$ to $X$, is stable with respect to random operator $T$.

\section{Acknowledgment}

This work was completed while the second author was visiting the Centre for Advanced Studies in Mathematics, Lahore University of Management Sciences, as a Research Associate.

\section{References}

[1] J.-P. Aubin and H. Frankowska, Set-Valued Analysis, Systems \& Control: Foundations \& Applications, vol. 2, Birkhäuser Boston, Massachusetts, 1990.

[2] I. Beg, Approximation of random fixed points in normed spaces, Nonlinear Analysis. Theory, Methods \& Applications. An International Multidisciplinary Journal. Series A: Theory and Methods 51 (2002), no. 8, 1363-1372.

[3] _ Minimal displacement of random variables under Lipschitz random maps, Topological Methods in Nonlinear Analysis 19 (2002), no. 2, 391-397.

[4] I. Beg and N. Shahzad, Random fixed points of random multivalued operators on Polish spaces, Nonlinear Analysis. Theory, Methods \& Applications. An International Multidisciplinary Journal. Series A: Theory and Methods 20 (1993), no. 7, 835-847.

[5] __ Applications of the proximity map to random fixed point theorems in Hilbert spaces, Journal of Mathematical Analysis and Applications 196 (1995), no. 2, 606-613.

[6] A. T. Bharucha-Reid, Fixed point theorems in probabilistic analysis, American Mathematical Society. Bulletin. New Series 82 (1976), no. 5, 641-657.

[7] C. E. Chidume, Approximation of fixed points of strongly pseudocontractive mappings, Proceedings of the American Mathematical Society 120 (1994), no. 2, 545-551.

[8] - Iterative solutions of nonlinear equations in smooth Banach spaces, Nonlinear Analysis. Theory, Methods \& Applications. An International Multidisciplinary Journal. Series A: Theory and Methods 26 (1996), no. 11, 1823-1834.

[9] J. A. Gatica and W. A. Kirk, Fixed point theorems for Lipschitzian pseudo-contractive mappings, Proceedings of the American Mathematical Society 36 (1972), no. 1, 111-115.

[10] K. Goebel and W. A. Kirk, A fixed point theorem for asymptotically nonexpansive mappings, Proceedings of the American Mathematical Society 35 (1972), 171-174.

[11] J. Górnicki, Weak convergence theorems for asymptotically nonexpansive mappings in uniformly convex Banach spaces, Commentationes Mathematicae Universitatis Carolinae 30 (1989), no. 2, 249-252.

[12] O. Hanš, Reduzierende zufällige transformationen, Czechoslovak Mathematical Journal 7(82) (1957), 154-158 (German), with Russian summary.

[13] _ Random operator equations, Proceedings of 4th Berkeley Sympos. Math. Statist. and Prob., Vol. II, University of California Press, California, 1961, pp. 185-202, part I.

[14] S. Itoh, Random fixed-point theorems with an application to random differential equations in $\mathrm{Ba}$ nach spaces, Journal of Mathematical Analysis and Applications 67 (1979), no. 2, 261-273. 
[15] W. A. Kirk, Remarks on pseudo-contractive mappings, Proceedings of the American Mathematical Society 25 (1970), 820-823.

[16] T.-C. Lin, Random approximations and random fixed point theorems for continuous 1-setcontractive random maps, Proceedings of the American Mathematical Society 123 (1995), no. 4, 1167-1176.

[17] Q. Liu, Iterative sequences for asymptotically quasi-nonexpansive mappings, Journal of Mathematical Analysis and Applications 259 (2001), no. 1, 1-7.

[18] Z. Opial, Weak convergence of the sequence of successive approximations for nonexpansive mappings, American Mathematical Society. Bulletin. New Series 73 (1967), 591-597.

[19] D. O'Regan, A continuation type result for random operators, Proceedings of the American Mathematical Society 126 (1998), no. 7, 1963-1971.

[20] N. S. Papageorgiou, Random fixed point theorems for measurable multifunctions in Banach spaces, Proceedings of the American Mathematical Society 97 (1986), no. 3, 507-514.

[21] J. A. Park, Mann-iteration process for the fixed point of strictly pseudocontractive mapping in some Banach spaces, Journal of the Korean Mathematical Society 31 (1994), no. 3, 333-337.

[22] N. Shahzad, Random fixed points of $K$-set-and pseudo-contractive random maps, Nonlinear Analysis. Theory, Methods \& Applications. An International Multidisciplinary Journal. Series A: Theory and Methods 57 (2004), no. 2, 173-181.

[23] S. P. Singh and B. Watson, Proximity maps and fixed points, Journal of Approximation Theory 39 (1983), no. 1, 72-76.

[24] A. Špaček, Zufällige Gleichungen, Czechoslovak Mathematical Journal 5(80) (1955), 462-466 (German), with Russian summary.

[25] K.-K. Tan and H. K. Xu, Iterative solutions to nonlinear equations of strongly accretive operators in Banach spaces, Journal of Mathematical Analysis and Applications 178 (1993), no. 1, 9-21.

[26] K.-K. Tan and X.-Z. Yuan, Random fixed point theorems and approximation, Stochastic Analysis and Applications 15 (1997), no. 1, 103-123.

[27] X. L. Weng, Fixed point iteration for local strictly pseudo-contractive mapping, Proceedings of the American Mathematical Society 113 (1991), no. 3, 727-731.

[28] H. K. Xu, Random fixed point theorems for nonlinear uniformly Lipschitzian mappings, Nonlinear Analysis. Theory, Methods \& Applications. An International Multidisciplinary Journal. Series A: Theory and Methods 26 (1996), no. 7, 1301-1311.

[29] H. K. Xu and I. Beg, Measurability of fixed point sets of multivalued random operators, Journal of Mathematical Analysis and Applications 225 (1998), no. 1, 62-72.

Ismat Beg: Centre for Advanced Studies in Mathematics, School of Arts and Sciences, Lahore University of Management Sciences (LUMS), 54792 Lahore, Pakistan

E-mail address: ibeg@lums.edu.pk

Mujahid Abbas: Department of Mathematics, Government Post Graduate College,

Sahiwal, Pakistan

E-mail address: mujahid@lums.edu.pk 


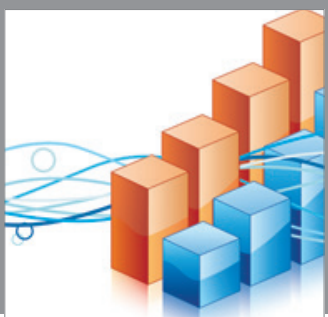

Advances in

Operations Research

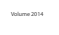

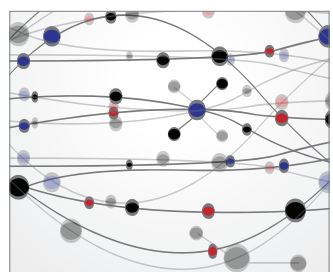

\section{The Scientific} World Journal
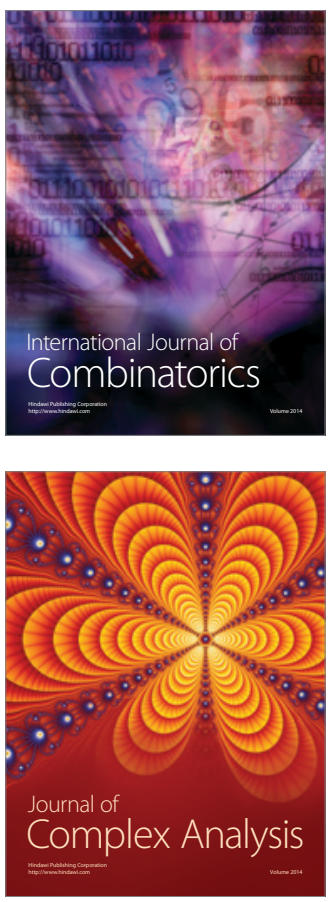

International Journal of

Mathematics and

Mathematical

Sciences
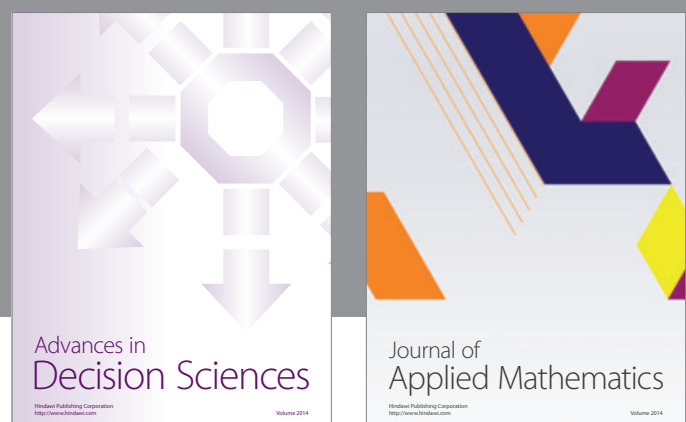

Journal of

Applied Mathematics
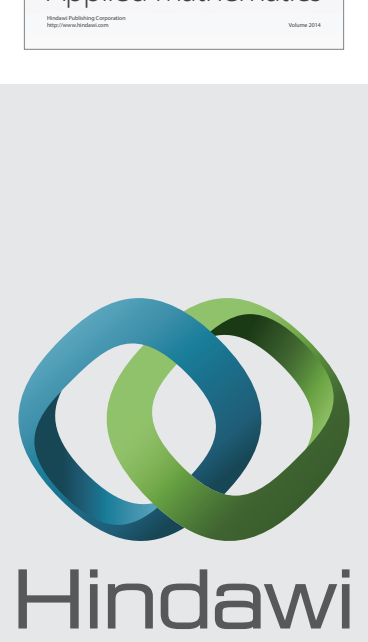

Submit your manuscripts at http://www.hindawi.com
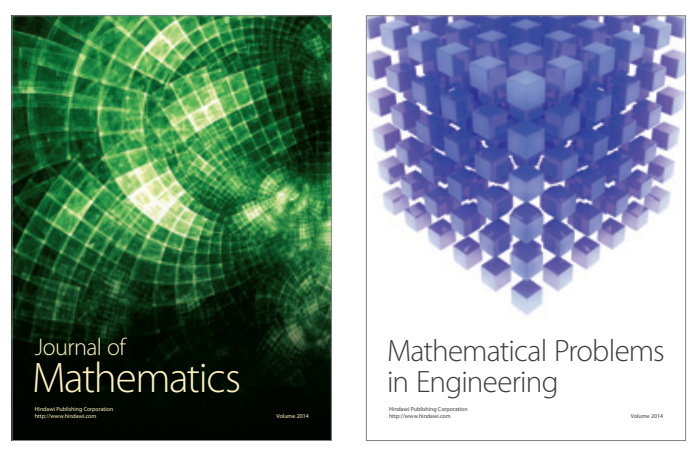

Mathematical Problems in Engineering
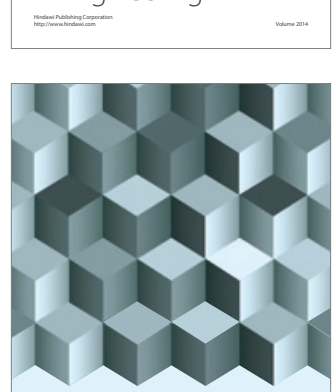

Journal of

Function Spaces
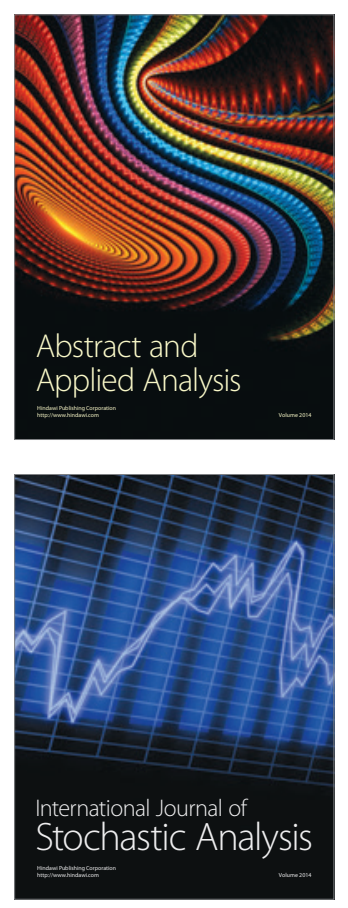

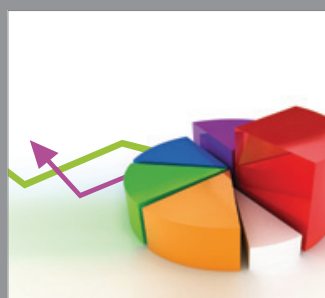

ournal of

Probability and Statistics

Promensencen
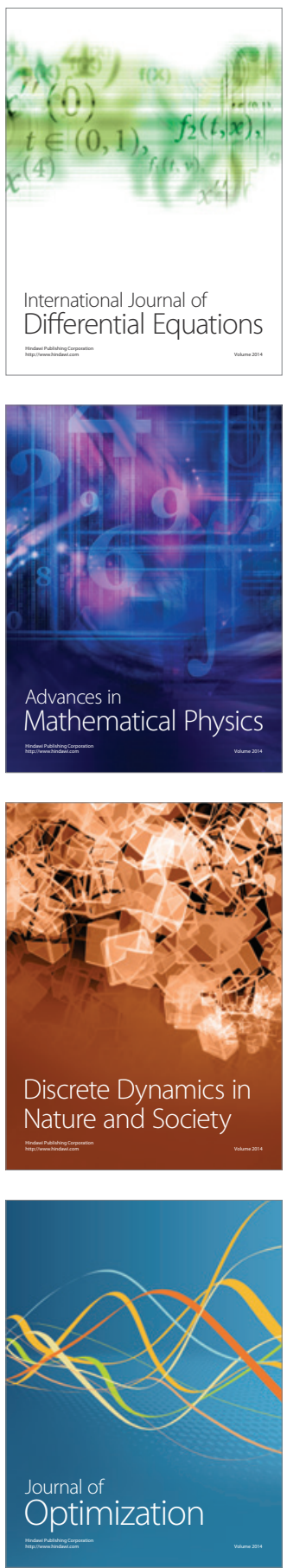